\title{
Experimental Test of the State Estimation-Reversal Tradeoff Relation in General Quantum Measurements
}

\author{
Geng Chen, Yang Zou, Xiao-Ye Xu, Jian-Shun Tang, Yu-Long Li, Jin-Shi Xu, Yong-Jian Han," \\ Chuan-Feng $\mathrm{Li}^{\dagger}{ }^{\dagger}$ and Guang-Can Guo \\ Key Laboratory of Quantum Information, University of Science and Technology of China, CAS, \\ Hefei 230026, China and Synergetic Innovation Center of Quantum Information and Quantum Physics, \\ University of Science and Technology of China, Hefei, Anhui 230026, China
}

\author{
Hai-Qiao Ni, ${ }^{2}$ Ying Yu, ${ }^{2}$ Mi-Feng $\mathrm{Li}^{2},{ }^{\text {Guo-Wei Zha, }}{ }^{2}$ and Zhi-Chuan $\mathrm{Niu}^{2}$ \\ National Laboratory for Superlattices and Microstructures, Institute of Semiconductors, \\ Chinese Academy of Sciences, P. O. Box 912, Beijing 100083, China
}

\author{
Yaron $\mathrm{Kedem}^{3}$ \\ Nordic Institute for Theoretical Physics (NORDITA), Roslagstullsbacken 23, S-106 91 Stockholm, Sweden
} (Received 13 December 2013; revised manuscript received 24 April 2014; published 5 June 2014)

\begin{abstract}
When a measurement has limited strength, only partial information, regarding the initial state, is extracted, and, correspondingly, there is a probability to reverse its effect on the system and retrieve the original state. Recently, a clear and direct quantitative description of this complementary relationship, in terms of a tradeoff relation, was developed by Y. K. Cheong and S. W. Lee. [Phys. Rev. Lett. 109, 150402 (2012)]. Here, this tradeoff relation is experimentally verified using polarization-encoded single photons from a quantum dot. Measurement operators representing a complete range, from not affecting the system to a projection to a single polarization state, are realized. In addition, for each measurement operator, an optimal reversal operator is also implemented. The upper bound of the tradeoff relation is mapped to experimental parameters representing the measurement strength. Our results complement the theoretical work and provide a hands-on characterization of general quantum measurements.
\end{abstract}

\section{INTRODUCTION}

The Heisenberg uncertainty principle (HUP) is one of the cornerstones of quantum mechanics [1,2]. It was originally discussed in the context of a measurement process, namely, a $\gamma$-ray microscope, and was perceived as a limitation on the possible precision that can be achieved. Nowadays, it is understood that the fundamental constraint refers to the intrinsic uncertainty of the quantum state and is independent of any measuring procedure [3]. Because of the considerable development in the quantum description of measurements, many limitations in the spirit of the HUP, or directly related to it, were considered. In the field of quantum metrology, the Heisenberg limit, where the precision scales as the number of interactions rather than the square root of it, is the aim of a wide theoretical [4,5] and experimental [6] effort. An extension, or an alternative

\footnotetext{
*smhan@ustc.edu.cn

†cfli@ustc.edu.cn
}

Published by the American Physical Society under the terms of the Creative Commons Attribution 3.0 License. Further distribution of this work must maintain attribution to the author(s) and the published article's title, journal citation, and DOI. formulation, to the HUP involving a quantification of measurement disturbance and its error was suggested [7], and its interpretations raised a lively debate [8] that involved some experimental results $[9,10]$. Inspired by the abstract ideas of complementary, or wave-particle, duality, and by defining concrete quantities such as visibility and distinguishability, some explicit (in)equalities were derived $[11,12]$ and later experimentally demonstrated $[13,14]$. The fact that these concepts, which were originally formulated as gedanken experiments, are being tested in labs, is remarkable, and it testifies to the way experiment and theory are pushing one another toward better understanding and better technological capabilities.

The vast research in the field of quantum information has resulted in many protocols for various tasks, such as communication, computation, or encryption. Naturally, the measurement process is a key ingredient in any such protocol, and an analysis of its properties is an important tool in assessing the reliability of any protocol. This motivation led to the development of several tradeoff relations regarding the degree of accuracy of joint quantum measurements, which can be seen as improved versions of the HUP [15-17]. Adapting an information-theoretic approach, different concepts related to precision and 
backaction were quantified, which were typically aimed at a specific task or protocol [18-23]. Recently, Cheong and Lee [24] derived such a tradeoff by considering nonprojective measurement. They related the general notions of precision and backaction with the well-defined quantities of estimation fidelity $G_{\max }$ and reversibility $P_{\text {rev }}$, respectively.

In order to define the estimation fidelity, consider that for every outcome $r$ of the measurement, the initial state $|\varphi\rangle$, which is unknown, is estimated by some state $\left|\tilde{\varphi}_{r}\right\rangle$. The fidelity of a specific estimation is given by $\left|\left\langle\tilde{\varphi}_{r} \mid \varphi\right\rangle\right|^{2}$, and the total fidelity of the process is given by averaging on all possible initial states

$$
G=\int d \varphi \sum_{r=1}^{N} p(r,|\varphi\rangle)\left|\left\langle\tilde{\varphi}_{r} \mid \varphi\right\rangle\right|^{2},
$$

where $N$ is the number of possible outcomes, $p(r,|\varphi\rangle)$ is the probability of getting an outcome $r$ when the initial state is $|\varphi\rangle$, and the integration is done according to the Haar measure. The value of $G$ depends on the strategy of selecting $\left|\tilde{\varphi}_{r}\right\rangle$, and the optimal case is denoted as $G_{\max }$.

For every outcome $r$, one can also attempt to retrieve the initial state $|\varphi\rangle$ from the postmeasurement state $\left|\varphi_{r}\right\rangle$ [25-29]. This reversal process might be done via some operator $\hat{R}^{(r)}$ that can be seen as an additional measurement, provided that $\hat{R}^{(r)}\left|\varphi_{r}\right\rangle \sim|\varphi\rangle$ up to some normalization. The success of this process would mean that the effect of the measurement is reversed, and the probability for that to happen is $\left|\left\langle\varphi\left|\hat{R}^{(r)}\right| \varphi_{r}\right\rangle\right|^{2}$. Given that the measurement operators are known, but neither the initial state $|\varphi\rangle$ nor, in general, even the postmeasurement state $\left|\tilde{\varphi}_{r}\right\rangle$ is known, one can choose the reversal operators $\hat{R}^{(r)}$, so as to maximize this probability. By averaging on all possible outcomes

$$
P_{\text {rev }}=\sum_{r=1}^{N}\left|\left\langle\varphi\left|\hat{R}^{(r)}\right| \varphi_{r}\right\rangle\right|^{2} p(r,|\varphi\rangle),
$$

we obtain the reversibility of the measurement.

The values of the estimation fidelity and the reversal probability depend on the measurement process and, more specifically, on its strength. Intuitively, one can say that the stronger the measurement is, $G$ becomes larger and $P_{\text {rev }}$ becomes smaller. Cheong and Lee have derived a strict tradeoff relation that, for the case of qubit (two-level) systems, can be expressed as

$$
6 G_{\mathrm{max}}+P_{\mathrm{rev}}=4 .
$$

We have performed an experiment to demonstrate the relation (3). By implementing the processes of a measurement with variable strength and an optimal probabilistic reversal to the initial state, we have measured directly the relevant quantities and confirmed the validity of the result.
In Sec. II, we discuss variable strength measurements and the optimal reversal process for a specific example, involving a single-qubit system. The relevant quantities are given explicit expressions in terms of experimental parameters. The details of the experimental setup are given in Sec. III. There, we describe how the measurement and reversal processes are realized and how $G_{\max }$ and $P_{\text {rev }}$ are extracted. Finally, in Sec. IV, the results are presented and discussed.

\section{MEASUREMENT AND REVERSAL OPERATIONS ON THE POLARIZATION OF A SINGLE PHOTON}

A general polarization state of a photon can be written as

$$
|\varphi\rangle=\sqrt{\alpha}|H\rangle+e^{i \phi} \sqrt{1-\alpha}|V\rangle,
$$

where $|H\rangle$ and $|V\rangle$ are the horizontal and vertical polarized states, respectively, $0 \leq \alpha \leq 1$, and $0 \leq \phi \leq 2 \pi$. The quantities of interest $G_{\max }$ and $P_{\text {rev }}$ involve an integration over the Hilbert space, which means preparing the system in the whole range of the parameters $\alpha$ and $\phi$. Because of our choice of measurement basis, the result is independent of $\phi$, so only the range of $\alpha$ is scanned.

Consider a general measurement with two possible outcomes, described by the operators

$$
\begin{gathered}
\hat{A}_{1}=\sqrt{1-\varepsilon}|H\rangle\langle H|+\sqrt{1-\eta}| V\rangle\langle V|, \\
\hat{A}_{2}=\sqrt{\varepsilon}|H\rangle\langle H|+\sqrt{\eta}| V\rangle\langle V|,
\end{gathered}
$$

where the values of $\varepsilon$ and $\eta$, ranging from 0 to 1 , are related to the strength of the measurements. We assume, without loss of generality, that $\varepsilon \leq \eta$. (The opposite case can be covered by relabeling the operators $\hat{A}_{1} \leftrightarrow \hat{A}_{2}$.) The (nonnormalized) postmeasurement states for outcomes 1 and 2 are given, respectively, by

$$
\begin{array}{r}
|\varphi\rangle_{1}=\sqrt{(1-\varepsilon) \alpha}|H\rangle+\sqrt{(1-\eta)(1-\alpha)} e^{i \phi}|V\rangle, \\
|\varphi\rangle_{2}=\sqrt{\varepsilon \alpha}|H\rangle+\sqrt{\eta(1-\alpha)} e^{i \phi}|V\rangle .
\end{array}
$$

The estimation fidelity is dependent on the strategy for guessing the state according to the measurement outcome. Here, we adopt the strategy introduced in Ref. [30], which has been proven to maximize the fidelity. For outcome 1 (2), we estimate the initial state to be $\left|\tilde{\varphi}_{1}\right\rangle=|H\rangle$ $\left(\left|\tilde{\varphi}_{2}\right\rangle=|V\rangle\right)$. For a certain state $|\varphi\rangle$, given by Eq. (4), the estimation fidelity is given by

$$
G_{\varphi}=\alpha^{2}(2 \eta-2 \varepsilon)+\alpha(1-3 \eta+\varepsilon)+\eta .
$$

Averaging over all possible states, we have 


$$
G_{\max }=\frac{1}{6}(3+\eta-\varepsilon) .
$$

From this expression, one can see that the minimum of $G_{\max }$ is $1 / 2$, which represents a random guess and corresponds to the case of $\eta=\varepsilon$, so the operators $\hat{A}_{1}$ and $\hat{A}_{2}$ are proportional to the identity matrix. The maximum value of $G_{\max }$ is $2 / 3$, which is a known bound [31].

To get the reversibility of a measurement, we should specify a strategy for the reversal process [32], i.e., choose operators $\hat{R}^{(r)}$, for each result $r$, that satisfy $\hat{R}^{(r)} \hat{A}_{r}=q \rrbracket$,

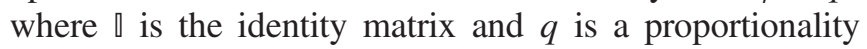
constant that we can choose. The choice of $q$ should maximize the probability of reversal $\left|\left\langle\varphi\left|\hat{R}^{(r)}\right| \varphi_{r}\right\rangle\right|^{2} \sim q^{2}$ while still satisfying the positive operator-valued measure condition $\left(\hat{R}^{(r)}\right)^{\dagger} \hat{R}^{(r)}+\left(\hat{F}^{(r)}\right)^{\dagger} \hat{F}^{(r)}=\rrbracket$, where $\hat{F}^{(r)}$ is the positive operator-valued measure element for the failure of the reversal procedure. Thus, the maximum value for $q$ is when the highest eigenvalue of $\hat{R}^{(r)}$ is 1 , and they can be written as

$$
\begin{gathered}
\hat{R}^{(1)}=\sqrt{\frac{1-\eta}{1-\varepsilon}}|H\rangle\langle H|+| V\rangle\langle V|, \\
\hat{R}^{(2)}=|H\rangle\left\langle H\left|+\sqrt{\frac{\varepsilon}{\eta}}\right| V\right\rangle\langle V| .
\end{gathered}
$$

Inserting Eq. (9) into Eq. (2), we get

$$
P_{\mathrm{rev}}=1+\varepsilon-\eta,
$$

which ranges from 0 -when $\varepsilon=0, \eta=1$, and the measurement is projective-to 1 -when $\eta=\varepsilon$ and the measurement strength vanishes.

\section{EXPERIMENTAL SETUP}

The experimental setup is shown in Fig. 1. An InAs/ GaAs single quantum dot (SQD) placed in cryostat (Microstat HiResII, Oxford Instruments) is used as the single-photon source. The pump beam uses the 632.8-nm line of a cw He-Ne laser, which is focused on the sample through a $20 \times$ microscopic objective (WJ-1020, Pvidi Technology). The emission from the quantum dot is collected by a $50 \times$ microscopic objective (HX-1050, Pvidi Technology). The initial state of the single photons [Eq. (4)] is prepared by the polarizer and a half-wave plate (HWP) mounted in the programmable motor-driven rotation stage. The traversal of states is realized through a rotation of the HWP to 51 angles, which corresponds to $\alpha_{i}=\frac{i}{50}(i=0, \ldots, 50)$.

Both the measurement and the reversal process are realized by Sagnac interferometers (SIs), each consisting of a polarized beam splitter (PBS) and a HWP mounted in the programmable motor-driven rotation stage for each path. Upon entry, the PBS splits the polarization component to different paths (see the inset in Fig. 1). On the path of the $|H\rangle(|V\rangle)$ component, a HWP is placed with its optical axis in an angle such as to make a transformation $|H\rangle \rightarrow \sqrt{1-\varepsilon}|H\rangle+\sqrt{\varepsilon}|V\rangle(|V\rangle \rightarrow \sqrt{\eta}|H\rangle+\sqrt{1-\eta}|V\rangle)$. In the main exit port of the PBS, which is the one directed at the second SI, each path is projected into its original

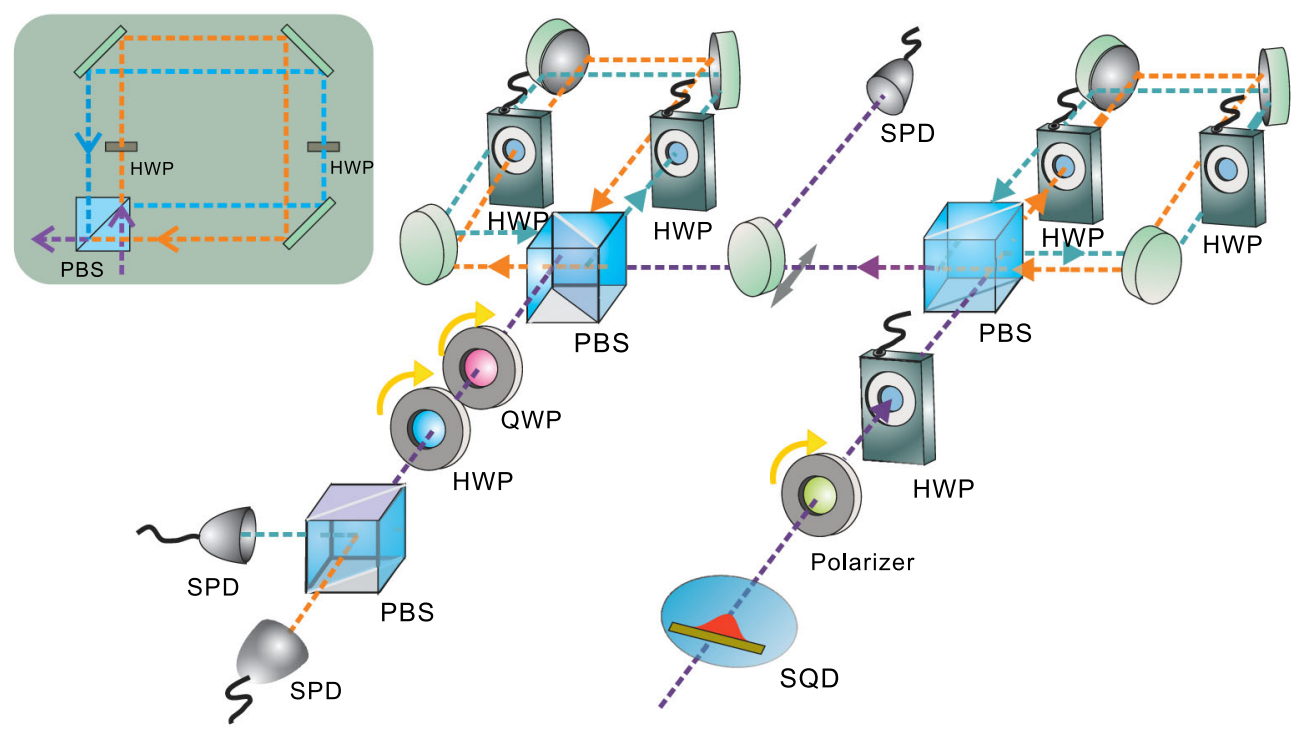

FIG. 1. Experimental setup for general measurement and reversal. Single photons from a quantum dot are prepared in a polarization state and sent through two Sagnac interferometers. The first one implements the measurement process via polarization-dependent modification of the state, and the second one reverses the operation. The result of the measurement is detected by inserting a mirror between the interferometers. A tomography process is performed on the photons coming out of the second interferometer. The inset is the planform of either SI. 
polarization and the components are joined. For an initial state [Eq. (4)], the outgoing state at this port is $\sqrt{(1-\varepsilon) \alpha}|H\rangle+\sqrt{(1-\eta)(1-\alpha)}|V\rangle$, which is a realization of $\hat{A}_{1}$. The state of a photon exiting on the other port, in the direction of the source, which is not monitored, corresponds to a measurement of $\hat{A}_{2}$. In order to implement $\hat{A}_{2}$, we set the angles of the HWP such that it will be realized in the main exit port.

In order to detect the measurement, a mirror is inserted between the two SIs, so as to direct the output of the first SI to a single-photon detector (SPD). We denote the count of this detector as $C_{\alpha}^{A 1(A 2)}(\varepsilon, \eta)$ when $\hat{A}_{1}\left(\hat{A}_{2}\right)$ is implemented.

The second SI is similar to the first one except that the angles of the HWPs are set to implement $\hat{R}^{(1)}$ or $\hat{R}^{(2)}$. A photon traveling through both SIs will have the same polarization as its initial state, regardless of the specific state. While there are some similarities between this process and the state-recovery process through the optical spin-echo effect [33], it is essentially different.

At the exit port of the second SI, a state tomography of the photons is implemented using a polarization analyzer, with a quarter-wave plate (QWP) and a HWP, a PBS, and two SPDs. The tomography of the retrieved state is done in order to confirm that the output state is identical to the initial one. The total counts in the two SPDs are denoted as $C_{\alpha}^{R 1}(\varepsilon, \eta)$ or $C_{\alpha}^{R 2}(\varepsilon, \eta)$ when $\hat{R}^{(1)}$ or $\hat{R}^{(2)}$ are implemented, respectively.

According to our guessing strategy, the fidelity is given by $\alpha$ for outcome 1 and $1-\alpha$ for outcome 2 . Each value should be multiplied by the number of times the outcome occurs, which is $C_{\alpha}^{A 1}(\varepsilon, \eta)$ or $C_{\alpha}^{A 2}(\varepsilon, \eta)$, and divided by the total number of measurements $C_{\alpha}^{A 1}(\varepsilon, \eta)+C_{\alpha}^{A 2}(\varepsilon, \eta)$. In addition, an average over the range of prepared states should be carried out. Thus, the value of $G_{\max }$ is

$$
G_{\max }=\frac{1}{51} \sum_{i=0}^{50} \frac{\alpha_{i} C_{\alpha_{i}}^{A 1}(\varepsilon, \eta)+\left(1-\alpha_{i}\right) C_{\alpha_{i}}^{A 2}(\varepsilon, \eta)}{C_{\alpha_{i}}^{A 1}(\varepsilon, \eta)+C_{\alpha_{i}}^{A 2}(\varepsilon, \eta)} .
$$

The reversibility is the probability to get a photon out of the second SI, so it is given by the counts in the last SPDs divided by the number of measurements and averaged over all states:

$$
P_{\mathrm{rev}}=\frac{1}{51} \sum_{i=0}^{50} \frac{C_{\alpha_{i}}^{R 1}(\varepsilon, \eta)+C_{\alpha_{i}}^{R 2}(\varepsilon, \eta)}{C_{\alpha_{i}}^{A 1}(\varepsilon, \eta)+C_{\alpha_{i}}^{A 2}(\varepsilon, \eta)} .
$$

\section{ANALYSIS OF THE EXPERIMENTAL RESULTS}

In the experiment, about $22 \mathrm{~K} / \mathrm{s}$ photons are collected and sent into the SIs, and the integration time for each data is $1 \mathrm{~s}$. The result [Eq. (3)] holds for any value of $\varepsilon$ and $\eta$. The involved quantities $G_{\max }$ and $P_{\text {rev }}$ depend on $\varepsilon$ and $\eta$

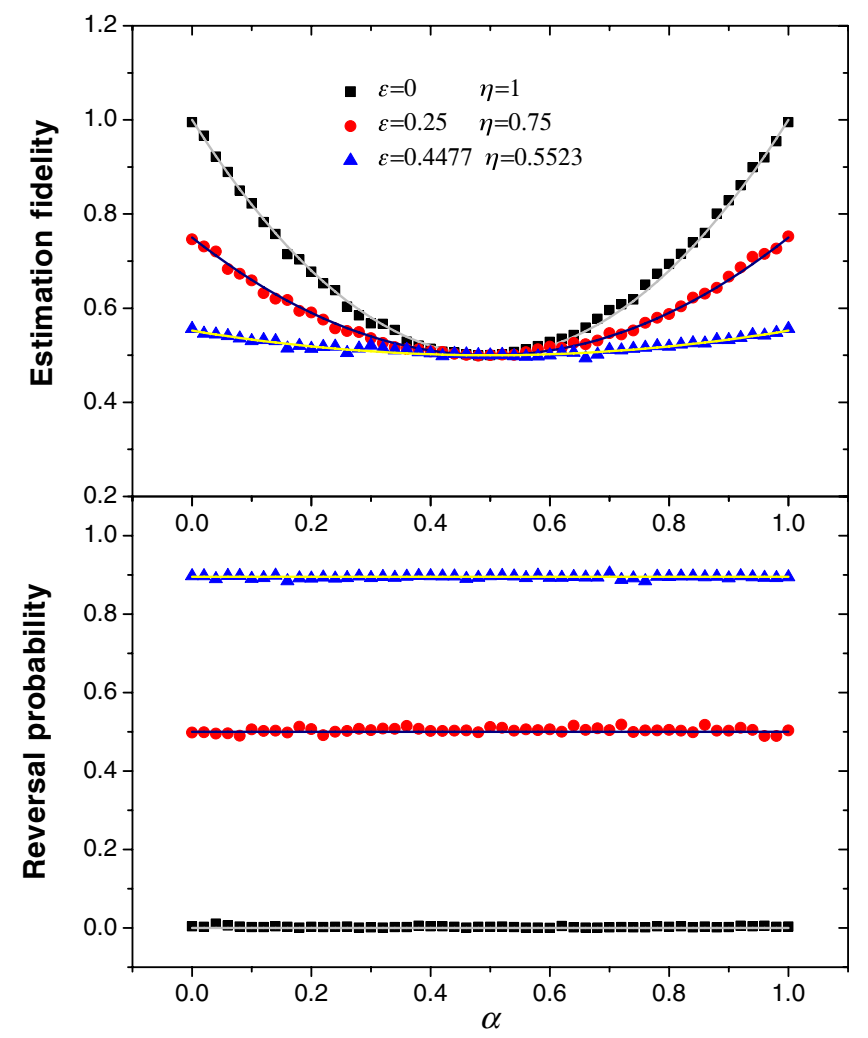

FIG. 2. Experimental results of the estimation fidelity and reversal probability for a specific state [Eq. (4)] described by $\alpha$. The estimation fidelity is minimal for the state with the highest uncertainty in the $H, V$ basis $\alpha=0.5$, while the reversal probability does not depend on the initial state. The error bars are smaller than the dimension of the markers and are not shown. The solid lines are the theoretical calculation.

and are defined via an integration over all possible states. Nonetheless, it can be instructive to see how the fidelity and reversibility for a given state [Eq. (4)] depend on its parameter $\alpha$. This dependency is shown in Fig. 2, for various values of $\varepsilon$ and $\eta$. When the state has low uncertainty, in the measurement basis, the estimation fidelity can be high and even approach 1 for strong measurement. The minimum value of $1 / 2$ is equivalent to a random guess and is independent of the state. Note that $G_{\max }$, which is an average over all states, cannot exceed $2 / 3$ [31].

The main focus of our experiment is to show the validity of the tradeoff result [Eq. (3)] which was derived in Ref. [24]. In the terms used here, our goal is to demonstrate how Eq. (3) holds for any choice of $\varepsilon$ and $\eta$. For this purpose, we have measured $G_{\max }$ and $P_{\text {rev }}$, via Eqs. (11) and (12), respectively, for 16 values of each parameter, ranging from 0 to 1 , i.e., 256 points in total. The experimental results are shown in Fig. 3, accompanied by a theoretical calculation. The diagonal line $\varepsilon=\eta$, where $G_{\max }$ is minimal and $P_{\text {rev }}$ is maximal, is a scenario of no measurement. The corners, where $|\varepsilon-\eta|=1$ and the 


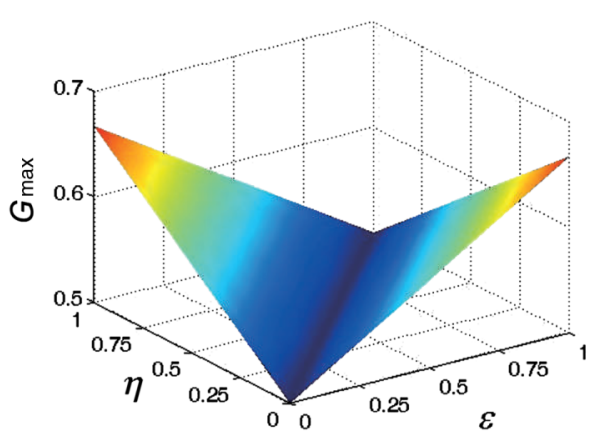

(a)

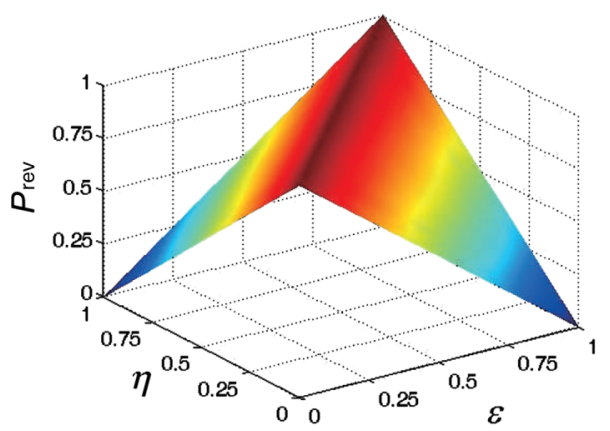

(c)

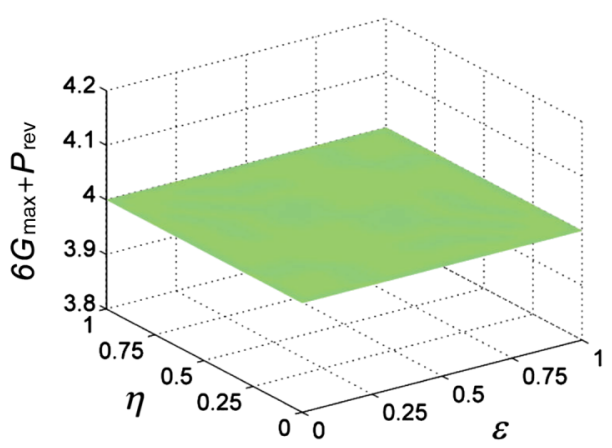

(e)

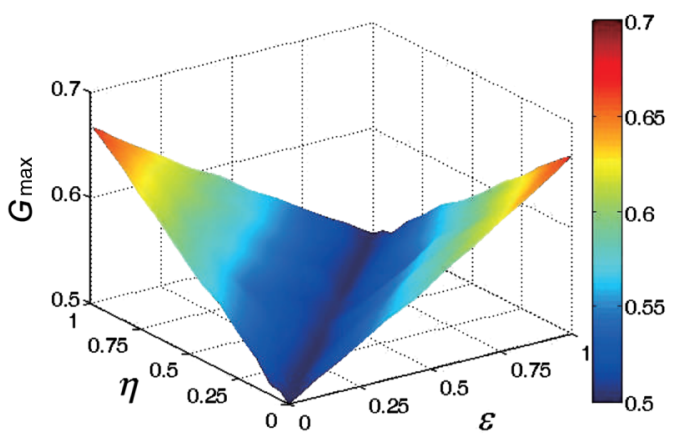

(b)

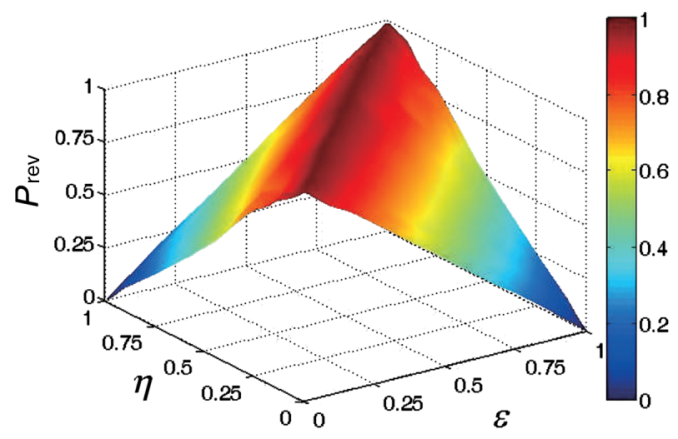

(d)

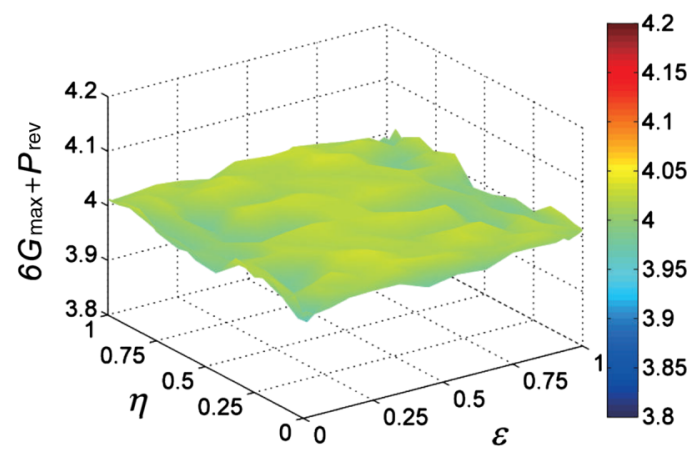

(f)

FIG. 3. A demonstration of the tradeoff relation (3): The experimental results of (b) $G_{\max }$ and (d) $P_{\text {rev }}$, as a function of the measurement parameters $\varepsilon$ and $\eta$ from 256 points, are shown along with the theoretical calculations (a) and (c), respectively. The different parameter regimes, which correspond to different measurement strengths, can be clearly identified via the magnitudes of these quantities. In addition, the sum $6 G_{\max }+P_{\text {rev }}$ is also shown, theoretically in (e) and the measurement results in (f). As required by Eq. (3), it is a plateau at 4. Note that the ratio between the scales in (b) and (d), or (a) and (c), is roughly 6, so the comparison, based on Eq. (3), is visible.

opposite extrema are shown, are the case of projective measurements. Although small fluctuations can be seen in Fig. 3(f), deviating from the theoretical value of 4, in each measurement point, the difference is within the error of the measurement. For example, the largest value is given by $4.026 \pm 0.031$. The errors are given by the standard deviation, where each measurement is performed 5 times. The main sources of the errors are the fluctuation of the SQD's emission, the uncertainties in aligning the wave plates, and the dark counts of the single-photon detectors.

In order to see that the state of the photons emitted from the second SI has been accurately reversed, quantum tomography is executed on a set of initial incoming states for fixed measurement parameters. The fidelity of the reversal process is shown in Fig. 4(a). For all of the 51 incoming states, this fidelity exceeds 0.99 , indicating a successful reversal process in the experiment. The high 

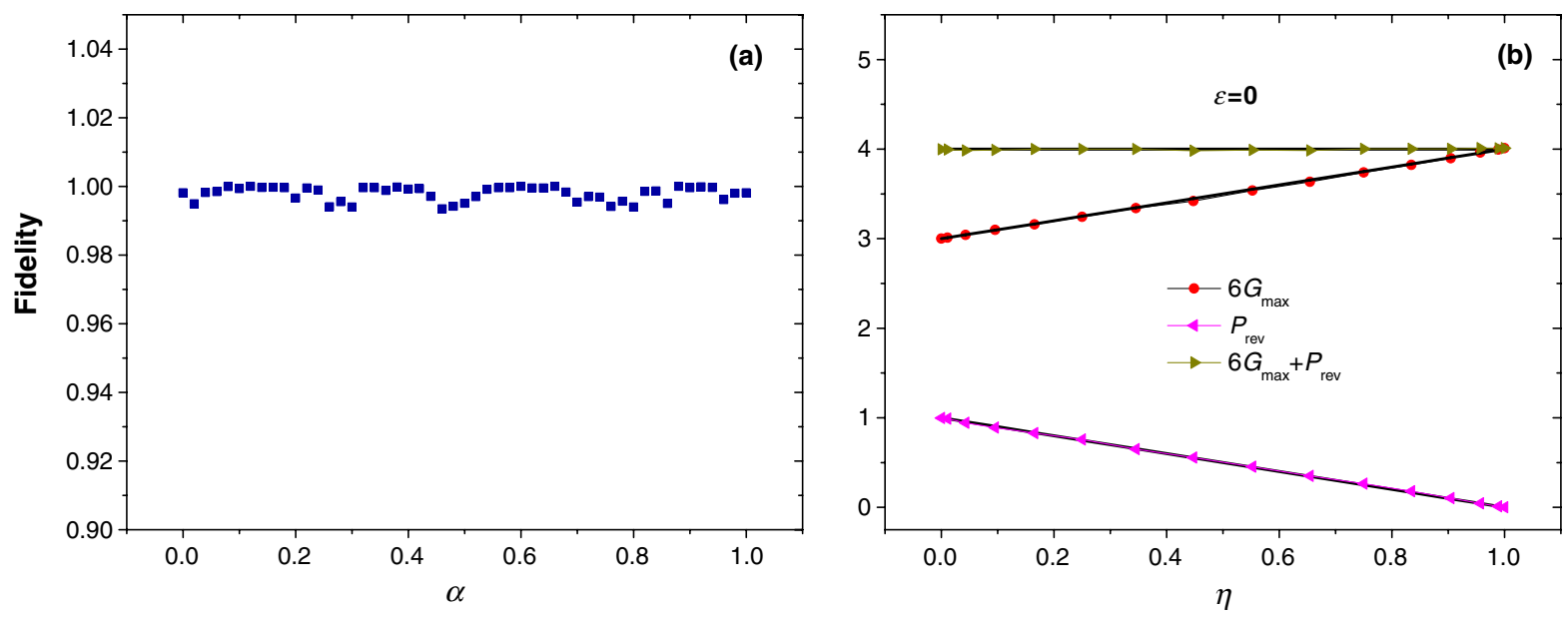

FIG. 4. (a) The fidelity of the reversal process: The overlap between the initial state that is prepared and the final state coming out of the second SI, where the tomography is preformed. The measurement parameters are $\varepsilon=0.25$ and $\eta=0.75$. (b) The relation between the measurement parameters and the measurement strength. The two terms on the left side of Eq. (3), $P_{\text {rev }}$ and $6 G_{\max }$, are shown separately, together with their sum. In order to show clearly the dependency on the measurement strength $|\varepsilon-\eta|$, we fix $\varepsilon=0$ while varying $\eta$ from 0 to 1 . The solid lines are the theoretical calculation.

reversal fidelity is due to the excellent quality of the two SIs, both of which exhibit high extinction ratios greater than 1000:1.

Examining the relation of the experimental parameters $\varepsilon$ and $\eta$ to the strength of the measurement might provide some insight. Intuitively, one can treat the difference $|\varepsilon-\eta|$ as the measurement strength. This view can be supported by looking on the extreme cases: For $\varepsilon=\eta$, both measurement operators $\hat{A}_{1}, \hat{A}_{2}$ are proportional to the identity matrix, so the state is not changed by the measurement and no information can be acquired. Since both parameters are in the range from 0 to 1 , the maximum of $|\varepsilon-\eta|$ is reached when one parameter is 0 and the other 1 . In this case, the measurement operators $\hat{A}_{1}, \hat{A}_{2}$ are simply projections and the measurement is projective. In order to substantiate this view, we look at the case of $\epsilon=0$, which means that $\eta$ represents the measurement strength. In Fig. 4(b), $6 G_{\max }+P_{\text {rev }}, 6 G_{\max }$, and $P_{\text {rev }}$ are plotted as functions of $\eta$. While $6 G_{\max }+P_{\text {rev }}$ remains constant, it is clearly shown how the estimation fidelity grows with $|\varepsilon-\eta|$, while the probability of reversal diminishes.

In summary, we experimentally tested the tradeoff relation concerning the balance between state estimation fidelity and reversibility in general quantum measurements on qubits. These quantities, which are well defined mathematically, were measured experimentally and were shown to obey the theoretical result [Eq. (3)] of Cheong and Lee [24]. The intuitive notion of measurement strength is connected to the experimental measurement parameters, and this connection is shown to be valid by examining the behavior of the estimation fidelity and the reversibility. Our results extend the theoretical work and provide a universal method to characterize general quantum measurements.

\section{ACKNOWLEDGMENTS}

This work was supported by the National Basic Research Program (No. 2011CB921200 and No. 2013CB933304), CAS, the National Natural Science Foundation of China (Grants No. 11274289, No. 11325419, No. 61327901, No. 90921015, No. 11105135, and No. 61308010), and the Fundamental Research Funds for the Central Universities (No. WK2470000011, No. WK2470000004, No. WK2470000006, and No. WJ2470000007). The work of Y.K. was supported in part by the ERC DM Grant No. 321031.

[1] W. Heisenberg, Uber den anschaulichen Inhalt der quantentheoretischen Kinematik und Mechanik, Z. Phys. 43, 172 (1927).

[2] E. H. Kennard, Zur Quantenmechanik einfacher Bewegungstypen, Z. Phys. 44, 326 (1927).

[3] J. Dressel and F. Nori, Certainty in Heisenberg's Uncertainty Principle: Revisiting Definitions for Estimation Errors and Disturbance, Phys. Rev. A 89, 022106 (2014).

[4] M. J. Holland and K. Burnett, Interferometric Detection of Optical Phase Shifts at the Heisenberg Limit, Phys. Rev. Lett. 71, 1355 (1993).

[5] M. Zwierz, C. A. Pérez-Delgado, and P. Kok, General Optimality of the Heisenberg Limit for Quantum Metrology, Phys. Rev. Lett. 105, 180402 (2010).

[6] V. Giovannetti and L. Maccone, Sub-Heisenberg Estimation Strategies Are Ineffective, Phys. Rev. Lett. 108, 210404 (2012).

[7] M. Ozawa, Universally Valid Reformulation of the Heisenberg Uncertainty Principle on Noise and Disturbance in Measurement, Phys. Rev. A 67, 042105 (2003). 
[8] P. Busch, P. Lahti, and R. F. Werner, Proof of Heisenberg's Error-Disturbance Relation, Phys. Rev. Lett. 111, 160405 (2013).

[9] L. A. Rozema, A. Darabi, D. H. Mahler, A. Hayat, Y. Soudagar, and A. M. Steinberg, Violation of Heisenberg's Measurement-Disturbance Relationship by Weak Measurements, Phys. Rev. Lett. 109, 100404 (2012).

[10] J. Erhart, S. Sponar, G. Sulyok, G. Badurek, M. Ozawa, and Y. Hasegawa, Experimental Demonstration of a Universally Valid Error-Disturbance Uncertainty Relation in Spin Measurements, Nat. Phys. 8, 185 (2012).

[11] G. Jaeger, A. Shimony, and L. Vaidman, Two Interferometric Complementarities, Phys. Rev. A 51, 54 (1995).

[12] B.-G. Englert, Fringe Visibility and Which-Way Information: An Inequality, Phys. Rev. Lett. 77, 2154 (1996).

[13] P. D. D. Schwindt, P. G. Kwiat, and B.-Gl. Englert, Quantitative Wave-Particle Duality and Nonerasing Quantum Erasure, Phys. Rev. A 60, 4285 (1999).

[14] G. J. Pryde, J. L. O’Brien, A. G. White, S. D. Bartlett, and T. C. Ralph, Measuring a Photonic Qubit without Destroying It, Phys. Rev. Lett. 92, 190402 (2004).

[15] M. M. Weston, M. J. W. Hall, M. S. Palsson, H. M. Wiseman, and G. J. Pryde, Experimental Test of Universal Complementarity Relations, Phys. Rev. Lett. 110, 220402 (2013).

[16] E. Arthurs and J. L. Kelly, On the Simultaneous Measurement of a Pair of Conjugate Observables, Bell Syst. Tech. J. 44, 725 (1965).

[17] E. Arthurs and M. S. Goodman, Quantum Correlations: A Generalized Heisenberg Uncertainty Relation, Phys. Rev. Lett. 60, 2447 (1988).

[18] F. Sciarrino, M. Ricci, F. De Martini, R. Filip and L. Mišta, Realization of a Minimal Disturbance Quantum Measurement, Phys. Rev. Lett. 96, 020408 (2006); M. F. Sacchi, Information-Disturbance Tradeoff in Estimating a Maximally Entangled State, Phys. Rev. Lett. 96, 220502 (2006).

[19] M. A. Nielsen and C. M. Caves, Reversible Quantum Operations and Their Application to Teleportation, Phys. Rev. A 55, 2547 (1997).
[20] S. L. Braunstein and A. K. Pati, Quantum Information Cannot Be Completely Hidden in Correlations: Implications for the Black-Hole Information Paradox, Phys. Rev. Lett. 98, 080502 (2007).

[21] F. Buscemi, M. Hayashi, and M. Horodecki, Global Information Balance in Quantum Measurements, Phys. Rev. Lett. 100, 210504 (2008).

[22] S. Luo, Information Conservation and Entropy Change in Quantum Measurements, Phys. Rev. A 82, 052103 (2010).

[23] H. Terashima, Information, Fidelity, and Reversibility in Single-Qubit Measurements, Phys. Rev. A 83, 032114 (2011).

[24] Y. K. Cheong and S. W. Lee, Balance between Information Gain and Reversibility in Weak Measurement, Phys. Rev. Lett. 109, 150402 (2012).

[25] M. Ueda and M. Kitagawa, Reversibility in Quantum Measurement Processes, Phys. Rev. Lett. 68, 3424 (1992).

[26] A. Royer, Reversible Quantum Measurements on a Spin 1/2 and Measuring the State of a Single System, Phys. Rev. Lett. 73, 913 (1994).

[27] M. Koashi and M. Ueda, Reversing Measurement and Probabilistic Quantum Error Correction, Phys. Rev. Lett. 82, 2598 (1999).

[28] A. N. Korotkov and A. N. Jordan, Undoing a Weak Quantum Measurement of a Solid-State Qubit, Phys. Rev. Lett. 97, 166805 (2006).

[29] N. Katz et al., Reversal of the Weak Measurement of a Quantum State in a Superconducting Phase Qubit, Phys. Rev. Lett. 101, 200401 (2008).

[30] Y.S. Kim, Y. W. Cho, Y. S. Ra, and Y. H. Kim, Reversing the Weak Quantum Measurement for a Photonic Qubit, Opt. Express 17, 11978 (2009).

[31] J. Preskill, Quantum Information and Computation, Lect. Notes Phys., http://www.theory.caltech.edu/people/preskill/ ph229/\#lecture.

[32] A. N. Jordan and A. N. Korotkov, Uncollapsing the Wavefunction by Undoing Quantum Measurements, Contemp. Phys. 51, 125 (2010).

[33] X. Y. Xu, J. S. Xu, C. F. Li, and G. C. Guo, MeasurementInduced Quantum Entanglement Recovery, Phys. Rev. A 82, 022324 (2010). 